\title{
Differenzierung von Nierentumoren mittels MRT
}

Hötker A et al. Differentiation of clear cell renal cell carcinoma from other renal cortical tumors by use of a quantitative multiparametric MRI approach. Am J Roentgenol 2017; 208: W85W91. doi:10.2214/AJR.16.16652

Nicht jeder mittels Bildgebung detektierte Nierentumor erweist sich nach der operativen Entfernung als bösartig. Bei manchen dieser Raumforderungen könnte auf eine Nephrektomie zugunsten einer weniger invasiven Behandlungsalternative verzichtet werden, wenn sich benigne und maligne Tumoren prätherapeutisch sicher voneinander unterscheiden ließen.

Wissenschaftler vom Memorial Sloan-Kettering Cancer Center in New York haben einen quantitativen, multiparametrischen, MR-basierten Diagnosealgorithmus entwickelt, mit dessen Hilfe dies möglich ist. Ihre Zielsetzung war es, insbesondere den aggressiven, prognostisch ungünstigen klarzelligen Subtyp des Nierenzellkarzinoms von anderen Raumforderungen zuverlässig abgrenzen zu können. Die Forscher haben retrospektiv die MR-Aufnahmen von 119 Patienten (71\% Männer), bei 
denen zwischen 2006 und 2013 ein Tumor der Nierenrinde neu diagnostiziert und mittels totaler oder partieller Nephrektomie behandelt worden war, ausgewertet. Insgesamt gingen 124 Raumforderungen in die Analyse ein. Die MR-Bildgebung umfasste diffusionsgewichtete (DWI), kontrastverstärkte sowie Chemical-Shift-Sequenzen. Zwei Radiologen werteten die Bilder unabhängig voneinander aus. Zunächst wurde das Tumorvolumen anhand aller verfügbaren Sequenzen bestimmt und voxelweise die durchschnittlichen apparenten Diffusionskoeffizienten (ADC) berechnet. Ferner wurden die Dynamik der Tumorkontrastierung (Upslope, Peak, Downslope, Area Under the Curve nach 100 s) sowie der „Chemical-Shift-Index“ berechnet. Als Referenz dienten die Ergebnisse der histopathologischen Untersuchung.

\section{Ergebnisse}

Bei 81 der 124 Tumoren (65,3\%) handelte es sich um klarzellige, bei 12 (9,7\%) um chromophobe, bei 12 (9,7\%) um papilläre und bei 8 (6,5\%) um nicht klassifizierte Nierenzellkarzinome. Weiterhin wurden 7 (5,6\%) Angiomyolipome und 4 (3,2\%) Onkozytome diagnostiziert. Die mediane Tumorgröße betrug insgesamt $7,16 \mathrm{~cm}$ (Variationsbreite 1-20,5), die der klarzelligen Nierenzellkarzinome $8 \mathrm{~cm}(1-16)$ und die der nicht klarzelligen Nierenzellkarzinome $4,5 \mathrm{~cm}(1,2-20,5)$. Die beiden befundenden Radiologen wiesen eine hohe Übereinstimmung auf. Die multivariate logistische Regressionsanalyse zeigte, dass sowohl der ADC als auch die maximale Kontrastierung (Peak Enhancement) und die Auswaschdynamik (Downslope) signifikant mit dem klarzelligen Nierenzellkarzinom assoziiert waren: Bei dieser aggressiven Tumorentität wurden bezüglich aller 3 Parameter signifikant höhere Werte nachgewiesen als bei den anderen Tumoren. Durch die Kombination der 3 Faktoren ließ sich die diagnostische Genauigkeit zusätzlich steigern. Es handelte sich mit hoher Wahrscheinlichkeit um ein klarzelliges Nierenzellkarzinom, wenn Folgendes zutraf:

\footnotetext{
- $1,011+3221 \times A D C+1,11 \times \log \quad$ (Peak Enhancement) $+3,220 \times$ Downslope $\geq 0,245$
}

Mithilfe dieser Methode erreichten die beiden Befunder bezüglich der Identifikation des klarzelligen Nierenzellkarzinoms eine Sensitivität von 0,897 bei einer Spezifität von 0,762 bzw. 0,738. Bei maximaler Sensitivität $(0,987)$ betrug die Spezifität 0,429 bzw. 0,262.

\section{FAZIT}

Mithilfe der multiparametrischen MRDiagnostik, so die Autoren, können klarzellige Nierenzellkarzinome nicht invasiv und sehr präzise von anderen renalen Tumoren unterschieden werden. Das Besondere des beschriebenen Ansatzes: Das gesamte Tumorvolumen wird analysiert, wodurch auch intratumorale Inhomogenitäten Berücksichtigung finden. Vielen älteren, multimorbiden Patienten mit einem als wenig aggressiv eingestuften Tumor könnte auf diese Weise ein risikoreicher operativer Eingriff erspart werden.

Dr. med. Judith Lorenz, Künzell 